\title{
Record Dynamics: Direct Experimental Evidence from Jammed Colloids
}

\author{
Dominic M. Robe ${ }^{1}$, Stefan Boettcher ${ }^{1}$, Paolo Sibani ${ }^{2}$, and Peter Yunker $^{3}$ \\ ${ }^{1}$ Department of Physics, Emory University, Atlanta, GA 30322, USA \\ ${ }^{2}$ FKF, Syddansk Universitet, DK5230 Odense M, Denmark and \\ ${ }^{3}$ School of Physics, Georgia Institute of Technology, Atlanta, GA 30332, USA
}

\begin{abstract}
In a broad class of complex materials a quench leads to a multi-scaled relaxation process known as aging. To explain its commonality and the astounding insensitivity to most microscopic details, record dynamics (RD) posits that a small set of increasingly rare and irreversible events, so called quakes, controls the dynamics. While key predictions of $\mathrm{RD}$ are known to concur with a number of experimental and simulational results, its basic assumption on the nature of quake statistics has proven extremely difficult to verify experimentally. The careful distinction of rare ("record") cage-breaking events from in-cage rattle accomplished in previous experiments on jammed colloids, enables us to extract the first direct experimental evidence for the fundamental hypothesis of RD that the rate of quakes decelerates with the inverse of the system age. The resulting description shows the predicted growth of the particle mean square displacement and of a mesoscopic lengthscale with the logarithm of time.
\end{abstract}

PACS numbers: 82.70.Dd, 05.40.-a, 64.70.pv

\section{INTRODUCTION}

Aging is a decelerating process present in manifold complex materials relaxing after a quench. It has been studied for decades from different perspectives [1 22, with the experimental focus moving, over the years, from relations such as the Fluctuation-Dissipation theorem and its violation [1, 2, on to the discovery, observation and study of the 'anomalous' non-equilibrium events 3 7. [1] now recognized as key properties. Concomitantly, theoretical and numerical studies have also considered anomalous events 8 10, and spin-glass thermoremanent magnetization [11, magnetic flux creep in type-II high- $T_{c}$ superconductors [12, ants moving out of their nest [16] and particle motion in dense colloids 20 22 have been interpreted using the statistics of 'quakes'. These increasingly rare cooperative changes are spatially localized within domains or clusters and lead the system from one metastable states to the next. The coarsegraining scheme referred to as 'record dynamics', links quakes with crossings of record high peaks in the freeenergy landscape [23, 24] associated to growing clusters and generated by the well-known exponential proliferation of local minima ('inherent states') with growing energy 25, 26. In dense colloidal suspensions, quakes are usually called 'cage breakings' while reversible quasiequilibrium (or Gaussian) fluctuations are called 'cage rattlings' 5, 27, 28.

Record dynamics has been proposed as a tool to model complex systems 24, 29 based on its ability to explain in a unified fashion the phenomenology of many different aging systems by filtering away all their microscopic peculiarities. The concurring experimental evidence pertains to macroscopic properties of magnetic glasses 9 11] and dense colloidal suspensions [20, 21. However, direct experimental support of its basic microscopic assumption is hard to gain and has been unavailable so far.

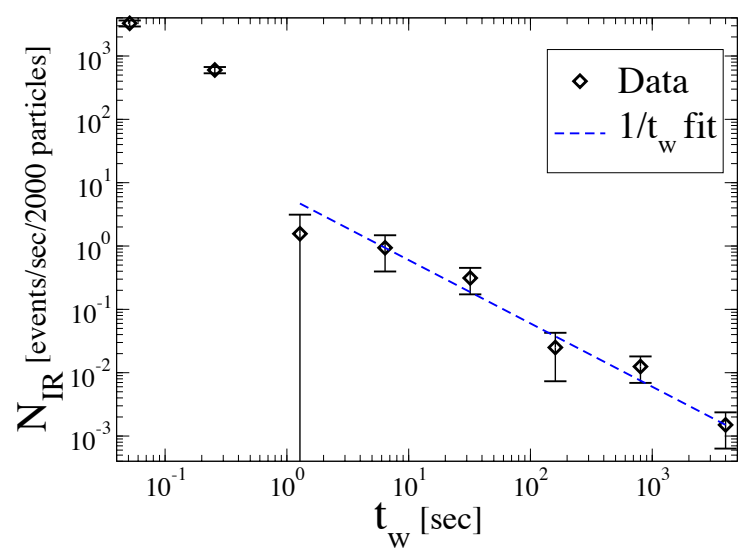

FIG. 1. Decay in the rate of intermittent cage-breaks ("quakes") in a dense colloid. Re-plot on log-scale of the experimental data in Fig. 2a of Yunker et al. [5]. The data directly validates the $1 / t$-decay fundamental to record dynamics that justifies the log-Poisson statistics [21, 22].

The data analyzed here stem from three experimental runs by Yunker et al [5] who employed an aqueous bidisperse suspension of micro-gel spheres sandwiched between glass cover slips to form a quasi-2D colloidal system with the unique property that a temperature change of $\approx 4 \mathrm{~K}$ at room temperature, could change the area fraction by $\approx 10 \%$ in $\approx 0.1 \mathrm{~s}$. The systems were prepared at a high packing fraction, heated uniformly by a mercury lamp to form a colloidal fluid, then quenched to form a glass by removing the light. Final area fractions studied were in the range $81-84 \%$. A window of 2500 particles, embedded in a sea of 300000 , was then tracked for $10^{4} \mathrm{~s}$. Most of the data presented here and in Ref. [5] stem from samples at the highest packing fractions and exhibit the strongest aging behavior, while our analysis of the loosest packings shows deviations from it. In Ref. [5], dynamic heterogeneity and aging were studied through analysis of 


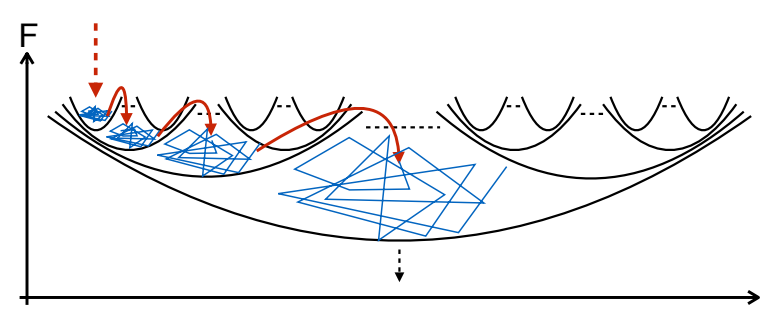

FIG. 2. Schematic view of the hierarchical free-energy landscape of a small jammed system (solid black), with a typical trajectory of an aging dynamics (blue and red). With increasing free energy $F$, local minima proliferate exponentially but also become shallower. The black-dashed down-arrow signifies that the hierarchy continues to a distant ground state. The dynamics generically evolves after a quench (red dashed arrow) through a sequence of quasi-equilibrium explorations (blue trajectories) and rare, intermittent quakes over record barriers (red arrows) that access an exponentially expanding portion of the configuration space.

changing particle neighborhoods. Here we use a threshold of $0.04 \mu \mathrm{m}$ to separate small reversible in-cage motion from irreversible displacements. Importantly, once irreversible configurational changes are identified, the significance of rare individual cage breaks for intermittency is demonstrated, and the connection between small and seemingly insignificant localized dislocations and much larger collective shifts in particle position is established.

These experiments provide the first opportunity to confront both the central assumption of record dynamics on quake statistics, as shown in Fig. 1. and one of its predictions, namely the emergence of growing mesoscopic real-space structures. In this Letter, we analyze the data of Ref. [5] to show that they concur with the fundamental assumptions as well as with the predictions of the theory. For the first time, direct experimental evidence for record dynamics as a coarse-grained description of dense colloids in particular, and of a broad class of aging materials in general, is provided.

\section{THEORETICAL BACKGROUND}

The relevance of records is immediate in the "Backgammon model" [30, in which $m$ particles are distributed over $n$ boxes $(m \gg n \gg 1)$ and where each update swaps one particle randomly between boxes. A box which empties out by a chance fluctuation of size $\sim m / n$ becomes inaccessible as the system's energy corresponds to the number of occupied boxes. The dynamics thus requires a sequence of rare record fluctuations of marginally increasing average size $\sim m / n, \sim m /(n-1)$, $\sim m /(n-2), \ldots$, to progress ever more slowly towards its ground state where all particles fit into one box. The importance of intermittent, irreversible record-events that are decorrelated by an exponential separation of timescales is obvious. In Ref. [22], a simple lattice model was introduced where mobile particles accrete into jammed clusters only to be re-mobilized after a chance fluctuation at a time exponential in the size of the cluster. Following a quench at $t=0$ when all particles are mobile, clusters form and break up ever more slowly because every break-up removes one cluster and increases the average size of the remaining clusters. We will use simulations of this model for comparisons with the experimental data. Finally, the connection between decelerating dynamics and growing mesoscopic real-space objects, our clusters or domains, is studied theoretically in Ref. 31, using a simple kinetically constrained model, the "parking lot model" [32, which has been applied in Ref. [33] to explain the logarithmically slow compactification of granular piles.

In disordered systems with a large number of degrees of freedom, the concept of "marginal attractor stability" first introduced in 34 is of central importance. In our context it presupposes three features that are wellestablished for the coarse-grained free-energy landscapes of complex systems, as illustrated in Fig. 2 (1) Metastable states with their combined basin of attraction, proliferate exponentially for increasing free energy [25, 26, 35, 36; (2) More-stable (lower-energy) states typically have higher barriers to escape their basin [36, 37]; and (3) overcoming increasingly higher barriers makes exponentially more configurations accessible [38]. The ensuing dynamics is also illustrated in Fig. 2. In a quench, the system almost certainly gets stuck initially in a shallow basin of high energy. There, a small, random fluctuation already suffices to escape into a larger basin containing many sub-basins, some of which feature local minima of lower energy. But for basins of lower energy, gradually higher fluctuations are required to escape. The gain in stability acquired in any one of these escapes will most probably only be marginal, since spontaneously arriving at minima of dramatically lower energy would be exponentially unlikely. In such a marginally deeper basin, the motion in and out of states less stable than the original basin only provides reversible quasi-equilibrium fluctuations. For an irreversible quake, typically, merely a record fluctuation in the random noise impinging on the system is required [39. In a spin glass a recordhigh thermal energy fluctuation elicits a quake associated with a large decrease in energy [8]. In a colloidal glass, record-sized fluctuations of locally available free space are needed to accommodate a new particle, and the final effect of a quake is a density increase.

In this manner, on exponentially longer timescales, ever larger and rare fluctuations become possible such that incrementally higher barriers can be scaled in a sequence of intermittent record-sized events, thereby making an exponentially growing number of configurations accessible. This approximation supersedes the particular properties of the hierarchies of barriers in a given system. As long as such a hierarchy exists, i.e., the system is actually jammed, these differences only vary the overall 
unit of time. To summarize, if barrier heights only grow marginally from one quake to the next, quake statistics can be meaningfully approximated by the statistics of record sized fluctuations in the random white noise which drives the dynamics [39]. Record dynamics thereby offers an analytical in-road to coarse-grained descriptions able to straddle the aging phenomenology.

\section{RESULTS FROM EXPERIMENTAL DATA}

\section{A. Statistics of Record-Sized Events}

The first key property of the experimental data of Ref. [5] that we check is that irreversible quakes are generated at a decelerating rate $\lambda(t) \propto 1 / t$, like records in any iid sequence of independent random numbers. Such events were identified as movements of particles involving a replacement of at least three nearest neighbors, shown in Fig. 2a of Ref. [5]. In our Fig. 1, we bin the same data logarithmically and see that, over more than three decades, the rate of irreversible events decelerates as $1 / t_{\mathrm{w}}$, with time $t_{\mathrm{w}}$ elapsed after the quench. Only an initial period of $\sim 0.1 \mathrm{~s}$, consistent with the time needed for the quench, shows significant deviations from the recorddynamics prediction. To obtain the data, Yunker et al. [5] had to filter out the "in-cage rattle", apparent from the inset of their Fig. 2a. This point is also emphasized by their Fig. 2b, that shows that a slow but steady increase of the domain-size of correlated events can be detected if reversible fluctuations are removed. Unfortunately, the sub-linear but perceptible growth in the relevant regime, i.e., for times $>0.1 \mathrm{~s}$, can not conclusively shown to be logarithmic with time, as record dynamics would predict 21, 22].

\section{B. Mean-Square Displacement}

As significant particle motion is activated by these quake events, the distance traveled is proportional to the integral of the rate $\lambda(t) \sim 1 / t$. Thus, the mean square displacement (MSD) of particles moving between times $t_{\mathrm{w}}$ and $t \geq t_{\mathrm{w}}$ in a dense colloid is predicted by record dynamics [21, 24] to grow as $\left\langle\Delta x^{2}\left(t, t_{\mathrm{w}}\right)\right\rangle \propto \ln \left(t / t_{\mathrm{w}}\right)$, where both time-arguments are counted from the initial quench. This 'logarithmic diffusion' was observed in Ref. 21] using particle track data by Courtland and Weeks [20] and is present in the cluster model discussed in Ref. 22]. However, tradition dictates that colloidal data be plotted versus the time-lag $\Delta t=t-t_{\mathrm{w}}$. Using this variable, one easily obtains $\left\langle\Delta x^{2}(\Delta t)\right\rangle \propto \ln \left(1+\Delta t / t_{\mathrm{w}}\right)$. Hence, as a function of $\Delta t$, the MSD grows linearly as $\sim \Delta t / t_{\mathrm{w}} \ll 1$ and as $\sim-\ln t_{\mathrm{w}}+\ln \Delta t$ for $\Delta t \gg t_{\mathrm{w}}$. In Fig. 3. we observe this behavior in the particle tracking data of Ref. 5 at the highest area fractions, $84 \%$ and $82 \%$, deep in the jammed phase. The left-hand panel shows that the MSD grows initially weakly as $\sim \Delta t / t_{\mathrm{w}}$, then crosses over for $\Delta t \gg t_{\mathrm{w}}$ to the predicted logarithmic growth. The right-hand panel of Fig. 3 shows that the MSD data for different $t_{\mathrm{w}}$ and $t$ can, indeed, be collapsed by plotting them as functions of $t / t_{\mathrm{w}}$. As we show further below, the collapse with $t / t_{\mathrm{w}}$ remains valid at other jammed packing fractions while the logarithmic slope of the plot increases with decreasing packing fraction. Only at densities too close to unjamming does the collapse begin to fail.

\section{Persistence}

Not considered in Ref. [5] is the decay of the persistence, i.e., the probability that a particle does not experience irreversible motion between times $t_{\mathrm{w}}$ and $t$. This quantity gives a clear picture of the spatial heterogeneity of glassy systems: If particle motion follows a regular Poisson process, the persistence decays exponentially in $\Delta t=t-t_{\mathrm{w}}$. In a log-Poisson process, this becomes an exponential in $\ln \left(t / t_{\mathrm{w}}\right)$, leading to a power-law decay in $t / t_{\mathrm{w}}$. This prediction was already borne out by simulations of jammed Lennard-Jones systems [40, plotted as a function of lag-time $\Delta t$ for different waiting times $t_{\mathrm{w}}$. There, after an initial plateau for $\Delta t \ll t_{\mathrm{w}}$, the data decays as a power law for $\Delta t \gg t_{\mathrm{w}}$. Analogous data from cluster-model simulations 22. have reproduced this behavior from the record dynamics and demonstrated a successful collapse of the data as a function $t / t_{\mathrm{w}}$.

In the lower panels of Fig. 4 , we show the experimental persistence data, plotted as functions of both, the lag time and $t / t_{\mathrm{w}}$, for several system ages $t_{\mathrm{w}}$. Here, a threshold of $0.04 \mu \mathrm{m}$ was used to separate reversible incage motion from a significant displacement after $t_{\mathrm{w}}$. As for MSD in Fig. 3, at the highest area fractions, $84 \%$ and $82 \%$, the data for the jammed system collapses on a $t / t_{\mathrm{w}}$-scale, but here in form of the predicted power-law. Overall, the relaxation dynamics is faster for less-jammed systems, such as that at $81 \%$ area fraction shown in the lowest panels of Fig. 4 However, the persistence collapse does not break down as much at the packing fraction near unjamming when compared to the MSD. This might reflect the fact that supercooled systems already exhibit considerable heterogeneity [27].

\section{Mobility Correlations}

In Ref. [5], the size of rearrangement events was characterized by the number of contiguous particles with unusually high speeds. While this approach is reasonable and common for detecting the presence of dynamic heterogeneity in tracking experiments, more precision is required to verify if the growth of event sizes with age corresponds to record dynamics. Therefore, the mobilitymobility correlation function $\chi_{4}$, widely used for supercooled liquids [41 43, has been proposed for this pur- 


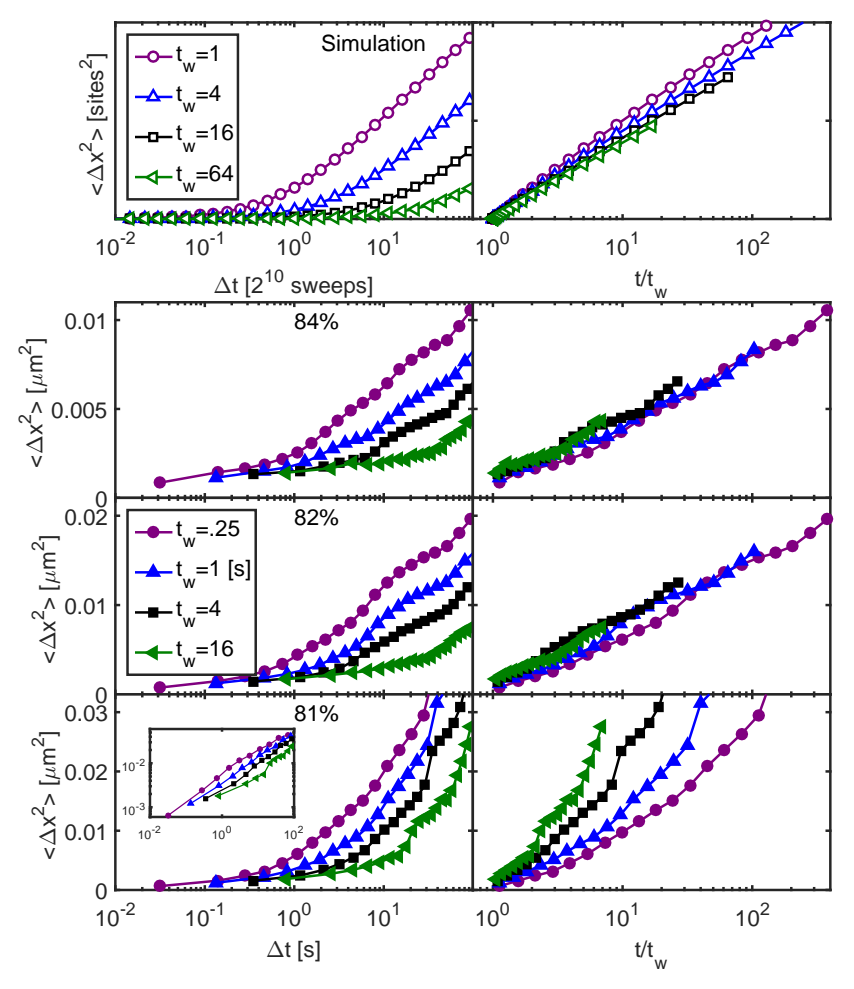

FIG. 3. Mean square displacement in simulations and in the experiments [5] on aging colloids at several area fractions. Panels of the same row contain the exact same data, however, each panel in the left column is plotted against the conventional lag-time $\Delta t=t-t_{\mathrm{w}}$, and against the logarithmic time-ratio $\ln \left(t / t_{\mathrm{w}}\right)$ suggested by record dynamics in the right column. The top row shows the simulation data, and the bottom three rows are experimental data arranged by decreasing area fractions, with $\approx 84 \%, \approx 82 \%$, and $\approx 81 \%$, from top to bottom. Time units for simulation data are in multiples of $2^{10}$ Monte Carlo sweeps, to avoid transients after the quench in the early decades. The inset to the bottom left panel contains the same data as that panel, but plotted on a log-log scale.

pose [17, 22, 44]; it is calculated as the ensemble variance of the total mobility at two times:

$$
M\left(t, t_{\mathrm{w}}\right)=\sum_{i} \exp \left(-\left|\overrightarrow{\mathbf{r}}_{i}(t)-\overrightarrow{\mathbf{r}}_{i}\left(t_{\mathrm{w}}\right)\right| / d\right),
$$

where $d=0.04 \mu \mathrm{m}$ is a typical length scale to distinguish mobile from immobile particles, here taken to be the same to as the persistence threshold above. The four-point correlation function $\chi_{4}$ is then defined as

$$
\chi_{4}\left(t, t_{\mathrm{w}}\right)=\left\langle M\left(t, t_{\mathrm{w}}\right)^{2}\right\rangle-\left\langle M\left(t, t_{\mathrm{w}}\right)\right\rangle^{2} .
$$

It is an as-of-yet unconfirmed prediction of record dynamics that the peak of $\chi_{4}$ grows as $\log t_{\mathrm{w}}$ [22. Reasoning by analogy with the cluster model, particles that move at time $t_{\mathrm{w}}$ belong to collapsing clusters of average size $\sim \log t_{\mathrm{w}}$. The re-activation of those particles requires that a cluster of the same size is reformed and collapses. Such process requires a time-interval $\Delta t_{\text {peak }}$

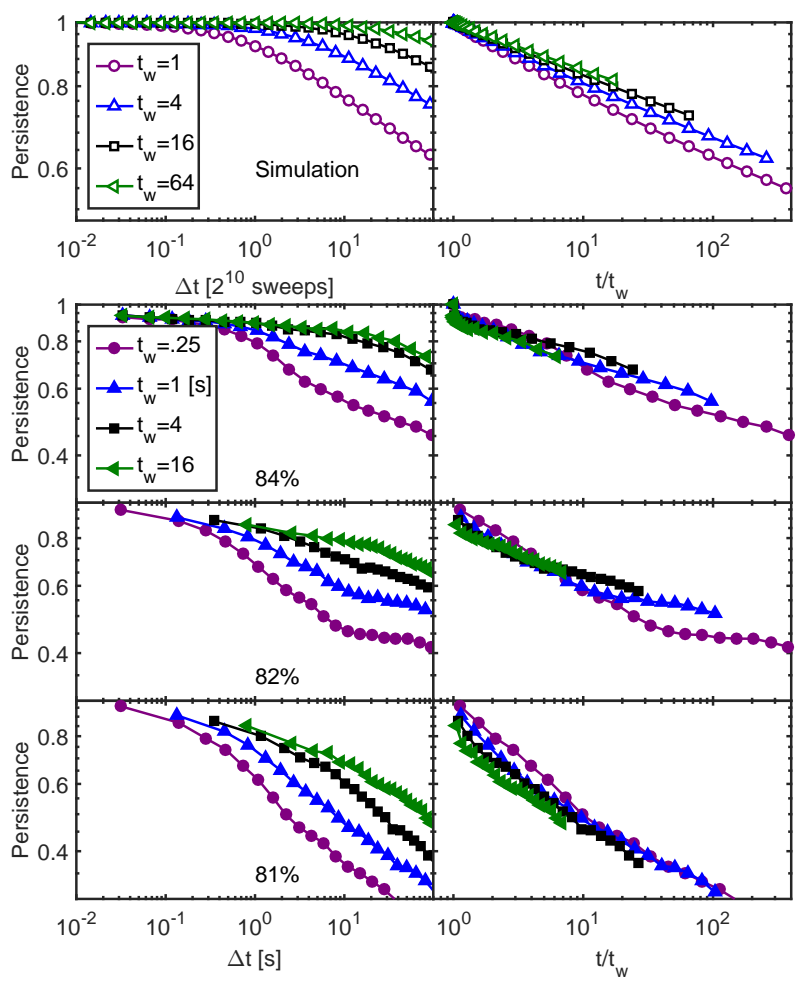

FIG. 4. Persistence in simulations and in the experiments [5] on aging colloids at several area fractions. The arrangement of panels is identical to that of Fig. 3

beyond $t_{\mathrm{w}}$ that grows monotonically with $t_{\mathrm{w}}$ : For times much shorter than $\Delta t_{\text {peak }}$, too few particles have a chance of reactivation, while for times much longer than $\Delta t_{\text {peak }}$ most of them already did re-activate and, hence, decorrelate from their mobility at $t_{\mathrm{w}}$. For the experimental data, consisting of single runs, there is no suitable ensemble to average over, so $\chi_{4}$ is approximated here by dividing a sample into four quadrants and taking the variance across those. This measure is plotted in Fig. 5 . There are large fluctuations in $\chi_{4}$ due to the unavoidable interdependence of the regions within a sample and the lack of statistics. In spite of this, the curves for different waiting times show regularly increasing peak heights for exponentially increasing waiting times, as predicted in simulations of record dynamics 22, also shown in Fig. 5 .

\section{E. Data at lower Area Fraction}

The data from Ref. [5] can be used to test the robustness of record dynamics over a range of area fractions. We have stated that specifics of a system do not impact the schematic description of the free-energy landscape in Fig. 2, as long as the system is jammed. Indeed, the experimental data for the fully jammed systems at the two area fractions $\approx 84 \%$ and $\approx 82 \%$ in Figs. 3.4 both show identical behavior for MSD as well as for persistence but for a different unit of time. Thus, only the 


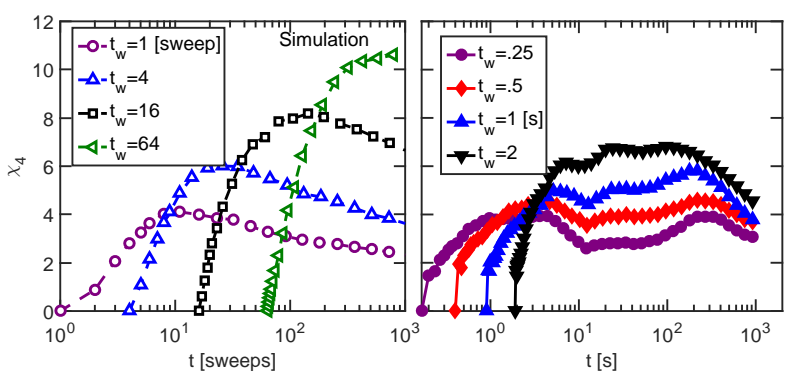

FIG. 5. Growth of length scale of dynamic heterogeneity as measured by the four-point susceptibility $\chi_{4}\left(t, t_{\mathrm{w}}\right)$, which is a measure of the mobile particles at time $t$ that had been mobile also at time $t_{\mathrm{w}}$. The height of each peak is proportional to the number of particles involved in a significant rearrangement event at time $t_{\mathrm{w}}$. Simulations of the cluster model 22 (left) have shown that the size of those events grows $\sim \ln t_{\mathrm{w}}$, reflected in the peak-height of $\chi_{4}$. The experimental data (right) shows a discernible peak growing monotonically with $t_{\mathrm{w}}$ in an approximately logarithmic manner consistent with the record dynamics prediction.

slopes for the respective collapse of the data is affected (note the scale on each y-axis), indicating less MSD and more persistence within a fixed amount of wall-time for denser systems.

The bottom panels of the same figures refer to a system at an area fraction of $\approx 81 \%$, whose behavior is consistent with being unjammed or being too near to the jamming transition. Thus, record dynamics is not expected to apply. In fact, the inset of the bottom left panel of Fig. 3 shows that the MSD exhibits nearly linear scaling vs. $\Delta t$, which is nearly diffusive behavior. Due to a weak $t_{\mathrm{w}}$ dependence, the MSD data do not collapse when plotted against $\ln \left(t / t_{\mathrm{w}}\right)$ in the rightmost panel. However, the data collapse in the bottom panels of Fig. 4 suggests that a semblance of dynamic heterogeneity is retained near the jamming transition in the persistence data.

\section{COMPARISON WITH SIMULATIONS OF THE CLUSTER MODEL}

Becker et al 22] simulated aging over 15 decades using a real-space implementation of record dynamics called the 'cluster model'. In this model, particles fill a lattice where in-cage rattle is explicitly course-grained away. All particles are assigned to contiguous clusters. There is a chance for a cluster to 'break up', which is exponentially unlikely in the size of the cluster. When this happens the cluster's constituent particles move minutely, and neighboring clusters spread into the broken cluster's territory. This process yields a breakup event rate proportional to $1 / t$ and an average cluster size proportional to $\ln t$. The $1 / t$ event rate is observed in the experimental data in Fig. 1. The growth of cluster size is a prediction of record dynamics that is consistent with observations of cluster sizes of fast particle in Fig. 2b in Ref. 5.
Here, the mean square displacement and persistence curves obtained from simulations are restricted to 3 decades of scaling to match the range of the experiments. When plotted against lag-time, shown in the upper left panel of Fig. 3, the simulation MSD exhibits the commonly expected plateau for $\Delta t \ll t_{\mathrm{w}}$ and rise for $\Delta t \gg t_{\mathrm{w}}$. Averaged over so many runs that error bars become invisible, the simulation curves clearly demonstrate the logarithmic behavior expected from record dynamics. The plateaus in the experimental data at high area fractions in Fig. 3 have a slight slope $\sim \Delta t / t_{\mathrm{w}} \ll 1$ due to the in cage motion. Both the simulation and experimental data collapse to a uniform logarithmic growth at all $t_{\mathrm{w}}$ when plotted against $t / t_{\mathrm{w}}$.

Persistence in the simulation is calculated as the fraction of particles that have not moved to a different lattice site. As with the MSD, due to the lack of in-cage rattle, the systems appear frozen for lag-times shorter than the waiting time. The persistence data from simulations shown in the top row of Fig. 4 demonstrate the powerlaw decay predicted by record dynamics. For the experimental data, a suitable metric had to be determined to classify particles as persistent. It was found that setting a distance threshold and taking the fraction of particles that move beyond that distance from their position at $t_{\mathrm{w}}$, robustly yields results qualitatively similar to the simulation curves. The experimental persistence curves shown here are all produced using a threshold of $0.04 \mu \mathrm{m}$.

\section{CONCLUSIONS}

Our detailed analysis of the experimental particle tracking data of Yunker et al. [5] i) Shows that the rate of irreversible particle rearrangements falls off as the inverse of the system age; ii) Confirms [21] that the positional variance due to particle motion between $t_{\mathrm{w}}$ and $t$ grows as $\ln t / t_{\mathrm{w}}$, a diffusive behavior in the logarithm of time; iii) Shows that persistence data can be scaled in the same way, as function of $\left.t / t_{\mathrm{w}} ; i v\right)$ Shows that the peak of the four-point susceptibility $\chi_{4}\left(t, t_{\mathrm{w}}\right)$ grows in a way consistent with $\ln t_{\mathrm{w}} ; v$ ) Confirms that the range of applicability of record dynamics is, as predicted, limited to sufficiently dense colloidal systems. Finally, the analysis of the experimental data concurs simulational data from our 'cluster model' 21, 22.

The agreement between experimental data and record dynamics predictions for all packing fractions in the jammed regime (and only there) confirms that aging dynamics is controlled in each instance by a small set of active variables, which move intermittently in time. That such variables cluster in space is well known as spatial heterogeneity and can be seen in the data of Ref. [5]. Even though more accurate experimental measurement of $\chi_{4}$ are required to ascertain whether the clusters grow logarithmically in time, there is clear experimental evi- 
dence that they do grow.

Continuous Time Random Walks (CTRW) are most widely used to coarse-grain the dynamics of systems jumping from trap to trap via rare intermittent events, see e.g. 36. Confusingly, CTRW and RD predictions are partly overlapping, while their physical mechanisms are very different. Importantly, as one of us argued in Ref. 24], CTRW are for a number of reasons inadequate descriptions of aging. Here we can only add that the emergence of a growing physical length scale suffices to rule out a renewal process as the underlying mechanism for aging in dense colloids. Real-space mesoscopic objects whose lifetime grows exponentially with their size or, equivalently, whose characteristic length grows logarithmically in time, are arguably a key feature of offequilibrium glassy dynamics and deserve further experimental investigation.

The authors are indebted to the V. Kann Rasmussen Foundation for support. SB and DMR thank SDU for its hospitality. SB and DMR are further supported by the NSF through grant DMR-1207431. PY thanks A.G. Yodh, K.B. Aptowicz, and Z. Zhang for useful conversations relating to Ref. [5].

[1] L. Buisson, L. Bellon, and S. Ciliberto. J. Phys. Cond. Mat., 15:S1163-S1179, 2003.

[2] L. Buisson, S. Ciliberto and A. Garciamartin. Europhys. Lett., 63:603, 2003.

[3] H. Bissig, S. Romer, L. Cipelletti, V. Trappe, and P. Schurtenberger. Phys. Chem. Comm., 6:21-23, 2003.

[4] K Vollmayr-Lee. J. Chem. Phys., 121:4781-4794, 2004.

[5] P. Yunker, Z. Zhang, K. B. Aptowicz, and A. G. Yodh. Phys. Rev. Lett., 103:115701, 2009.

[6] Tadashi Kajiya, Tetsuharu Narita, Veronique Schmitt, Francois Lequeuxa, and Laurence Talini. Soft Matter, 9:11129, 2013.

[7] Rojman Zargar, Bernard Nienhuis, Peter Schall, and Daniel Bonn. Phys. Rev. Lett., 110:258301, 2013.

[8] P. Sibani and H. Jeldtoft Jensen. Europhys. Lett., 69:563569, 2005.

[9] A. Crisanti and F. Ritort. Europhys. Lett., 66:253-259, 2004.

[10] P. Sibani. Phys. Rev. E, 74:031115, 2006.

[11] P. Sibani, G.F. Rodriguez and G.G. Kenning. Phys. Rev. B, 74:224407, 2006.

[12] L. P. Oliveira, H. J. Jensen, M. Nicodemi, and P. Sibani. Phys. Rev. B, 71:104526, 2005.

[13] V.S. Zotev, G.F. Rodriguez, G.G. Kenning, R. Orbach, E. Vincent and J. Hammann. Phys. Rev. B, 67:184422, 2003.

[14] G. F. Rodriguez, G. G. Kenning, and R. Orbach. Phys. Rev. Lett., 91:037203, 2003.

[15] G. F. Rodriguez, G. G. Kenning, and R. Orbach. Effect of the thermal quench on aging in spin glasses. Phys. Rev. B, 88:054302, 2013.

[16] Thomas O. Richardson, Elva J. H. Robinson, Kim Christensen, Henrik J. Jensen, Nigel R. Franks, and Ana B.
Sendova-Franks. PLoS ONE, 5(3):1-7, 032010.

[17] Azita Parsaeian and Horacio E. Castillo. Phys. Rev. E, 78:060105R, 2008.

[18] L. A. Fernandez, V. Martin-Mayor, G. Parisi, and B. Seoane. Temperature chaos in 3d ising spin glasses is driven by rare events. EPL, 103(6):67003, 2013.

[19] Katharina Vollmayr-Lee, Christopher H. Gorman, and Horacio E. Castillo. Journal of Chemical Physics, 144:234510, 2016.

[20] Rachel E. Courtland and Eric R. Weeks. J. Phys.: Condens. Matter, 15:S359-S365, 2003.

[21] S. Boettcher and P. Sibani. Aging in dense colloids as diffusion in the logarithm of time. J. Phys.: Condens. Matter, 23:065103, 2011.

[22] Nikolaj Becker, Paolo Sibani, Stefan Boettcher, and Skanda Vivek. J. Phys.: Condens. Matter, 26:505102, 2014.

[23] Paolo Sibani and Karl Heinz Hoffmann. Phys. Rev. Lett., 63:2853-2856, 1989.

[24] Paolo Sibani. EPL, 101:30004, 2013.

[25] Frank H. Stillinger and Thomas A. Weber. Science, 225:983-989, 1984.

[26] Frank H. Stillinger. Phys. Rev. E, 59:48-51, 1999.

[27] Eric R. Weeks, J.C. Crocker, Andrew C. Levitt, Andrew Schofield, and D.A. Weitz. Science, 287:627-631, 2000.

[28] Gary L. Hunter and Eric R. Weeks. Rep. Prog. Phys., 75:066501, 2012.

[29] P. Anderson, H. J. Jensen, L. P. Oliveira, and P. Sibani. Complexity, 10:49-56, 2004.

[30] Felix Ritort. Physical Review Letters, 75:1190, 1995.

[31] Paolo Sibani and Stefan Boettcher. Phys. Rev. E, 93:062141, Jun 2016.

[32] P. L. Krapivsky and E. Ben-Naim. Collective properties of adsorption-desorption processes. J. Chem. Phys., 100:6778, 1994.

[33] Edmund R. Nowak, James B. Knight, Eli Ben-Naim, Heinrich M. Jaeger, and Sidney R. Nagel. Phys. Rev. E, 57:1971-1982, 1998.

[34] Chao Tang, Kurt Wiesenfeld, Per Bak, Susan Coppersmith and Peter Littlewood. Phys. Rev. Lett., 58:11611164, 1987.

[35] A J Bray and M A Moore. Journal of Physics C: Solid State Physics, 14:2629-2664, 1981.

[36] Andreas Heuer. J. Phys.: Condens. Matter, 20:373101, 2008.

[37] P. Sibani, J. C. Schön, P. Salamon, and J.-O. Andersson. Emergent hierarchical structures in complex-system dynamics. Europhys. Lett., 22:479, 1993.

[38] S. Boettcher and P. Sibani. Comparing extremal and thermal explorations of energy landscapes. Eur. Phys. J. B, 44:317-326, 2005.

[39] Paolo Sibani and Peter B. Littlewood. Phys. Rev. Lett., 71:1482, 1993.

[40] Djamel El Masri, Ludovic Berthier, and Luca Cipelletti. Phys. Rev. E, 82:031503, 2010.

[41] L. Berthier, G. Biroli, J.-P. Bouchaud, L. Cipelletti, D. ElMasri, D. L'Hote, F. Ladieu, and M. Pierno. Science, 310:1797, 2005.

[42] Ludovic Berthier, Giulio Biroli, Jean-Philippe Bouchaud, Luca Cipelletti, and Wim van Saarloos, editors. Oxford University Press, 2011.

[43] Ludovic Berthier. Physics, 4:42, 2011.

[44] C. Maggi, R. D. Leonardo, G. Ruocco, and J. C. Dyre. Phys. Rev. Lett., 109:097401, 2012. 